\title{
CHARACTERIZATION OF A CLASS OF FUNCTIONS USING DIVIDED DIFFERENCES
}

\author{
PLAMEN SIMEONOV
}

Received 5 April 2000

We determine the class of functions, the divided difference of which, at $n$ distinct numbers, is a continuous function of the product of these numbers.

\section{Introduction}

We first introduce the needed terminology. The divided differences of a function $f$ at distinct points are defined recursively as follows:

$$
\begin{gathered}
f\left[x_{1}\right]:=f\left(x_{1}\right), \\
f\left[x_{1}, \ldots, x_{n}\right]:=\frac{f\left[x_{2}, \ldots, x_{n}\right]-f\left[x_{1}, \ldots, x_{n-1}\right]}{x_{n}-x_{1}}, \quad n \geq 2 .
\end{gathered}
$$

The following formula is well known, [2],

$$
f\left[x_{1}, \ldots, x_{n}\right]=\sum_{j=1}^{n} \frac{f\left(x_{j}\right)}{\omega_{n}^{\prime}\left(x_{j}\right)},
$$

where $\left\{x_{j}\right\}_{j=1}^{n} \subset \mathbb{C}$ are distinct numbers and $\omega_{n}(x):=\prod_{k=1}^{n}\left(x-x_{k}\right)$.

Furthermore, if $f \in \mathscr{P}_{n-1}$, the set of algebraic polynomials of degree at most $n-1$, then, [2], $f\left[x_{1}, \ldots, x_{n+1}\right]=0$. This fact can be proved by induction in $n$.

The more general definition of divided differences allowing repeated points is that $f\left[x_{1}, \ldots, x_{n}\right]:=a_{n-1}$, where $p_{n-1}(x)=\sum_{k=0}^{n-1} a_{k} x^{k}$ is the polynomial that interpolates $f$ at $\left\{x_{j}\right\}_{j=1}^{n}$ in the Hermite sense, [2].

The main results of this paper are the following theorems.

THEOREM 1.1. Let $n \in \mathbb{N}, n \geq 2$, be fixed and $f$ be a real-valued function defined on an open set $D$ of the real line $\mathbb{R}$. Assume that the divided difference $f\left[x_{1}, \ldots, x_{n}\right]$ satisfies

Copyright $(9) 2000$ Hindawi Publishing Corporation

Abstract and Applied Analysis 5:2 (2000) 85-90

2000 Mathematics Subject Classification: 39A05, 39B05

URL: http://aaa.hindawi.com/volume-5/S1085337500000294.html 
the functional equation

$$
f\left[x_{1}, \ldots, x_{n}\right]=G\left(\prod_{j=1}^{n} x_{j}\right)
$$

for every set of $n$ distinct numbers $\left\{x_{j}\right\}_{j=1}^{n} \subset D$, and $G$ is a continuous function on the set of products $P_{n}(D):=\left\{\prod_{j=1}^{n} x_{j}:\left\{x_{j}\right\}_{j=1}^{n} \subset D\right\}$. Then

$$
f(x)=\frac{a_{-1}}{x}+\sum_{k=0}^{n-1} a_{k} x^{k} .
$$

Furthermore, $G(t)=(-1)^{n+1} a_{-1} / t+a_{n-1}$.

THeOREM 1.2. Let $f$ be a complex-valued function defined on an open set $D$ of the complex plane $\mathbb{C}$. Assume that $f$ satisfies the conditions of Theorem 1.1 on D. Then $f$ and $G$ have the forms given by Theorem 1.1.

A similar characterization was obtained in [1] for functions the divided difference of which at $n$ distinct points is a function of the sum of the points.

\section{Proofs of the theorems}

Proof of Theorem 1.1. First assume that $0 \notin D$. We claim that $f \in C^{\infty}(D)$, that is, $f$ has continuous derivatives of arbitrary order in $D$. Let $\left\{x_{j}\right\}_{j=1}^{n} \subset D$ be distinct. From (1.2) and (1.3) for $f\left[x_{1}, \ldots, x_{n}\right]$ we get

$$
\begin{aligned}
& \left(\frac{f\left(x_{2}\right)-f\left(x_{1}\right)}{x_{2}-x_{1}}\right)\left(\frac{x_{2}-x_{1}}{\omega_{n}^{\prime}\left(x_{2}\right)}\right) \\
& \quad+f\left(x_{1}\right)\left(\frac{1}{\omega_{n}^{\prime}\left(x_{2}\right)}+\frac{1}{\omega_{n}^{\prime}\left(x_{1}\right)}\right)+\sum_{j=3}^{n} \frac{f\left(x_{j}\right)}{\omega_{n}^{\prime}\left(x_{j}\right)}=G\left(x_{1} \cdots x_{n}\right) .
\end{aligned}
$$

We let $x_{2} \rightarrow x_{1}$ in (2.1). All terms on the left-hand side of (2.1) beginning with the second term which tends to $\left.f\left(x_{1}\right)\left(1 / \prod_{j=3}^{n}\left(x-x_{j}\right)\right)^{\prime}\right|_{x=x_{1}}$, and the right-hand side $G$ have finite limits. Then the first term on the left-hand side of (2.1) has a finite limit, that is, $f^{\prime}\left(x_{1}\right)$ exists. Since the points $x_{1}, x_{3}, \ldots, x_{n}$ are distinct, all terms in the equation obtained from (2.1) after taking the limit $x_{2} \rightarrow x_{1}$, except possibly the first one, are continuous at $x_{1}$ for fixed $\left\{x_{j}\right\}_{j=3}^{n}$. We obtain $f \in C^{1}(D)$. Then (1.2) and (1.3) imply $G \in C^{1}\left(P_{n}(D)\right)$. This can be seen by observing that the derivative of the right-hand side of (1.2) with respect to $x_{1}$ is continuous at $x_{1}$ if the points $\left\{x_{j}\right\}_{j=1}^{n}$ are distinct and $\left\{x_{j}\right\}_{j=2}^{n}$ are fixed. Then with $a=\prod_{j=2}^{n} x_{j}, d G\left(a x_{1}\right) / d x_{1}$ exists and is continuous at $x_{1}$. Therefore, $G^{\prime}(t) \in C\left(P_{n}(D)\right)$ exists because every $t \in P_{n}(D)$ can be written as a product of $n$ distinct numbers from $D$ and $P_{n}(D)$ is an open set. 
Next, from (1.2) we have

$$
\lambda^{n-1} f\left[\lambda x_{1}, \ldots, \lambda x_{n}\right]=\sum_{j=1}^{n} \frac{f\left(\lambda x_{j}\right)}{\omega_{n}^{\prime}\left(x_{j}\right)}=\lambda^{n-1} G\left(\lambda^{n} x_{1} \cdots x_{n}\right) .
$$

Differentiating (2.2) with respect to $\lambda$ and setting $\lambda=1$, we obtain

$$
\begin{aligned}
\left(x f^{\prime}(x)\right)\left[x_{1}, \ldots, x_{n}\right] & =\sum_{j=0}^{n} \frac{x_{j} f^{\prime}\left(x_{j}\right)}{\omega_{n}^{\prime}\left(x_{j}\right)} \\
& =(n-1) G\left(x_{1} \cdots x_{n}\right)+n x_{1} \cdots x_{n} G^{\prime}\left(x_{1} \cdots x_{n}\right) .
\end{aligned}
$$

Equation (2.3) for $f_{1}(x)=x f^{\prime}(x)$ has the same form as (1.3) for $f(x)$, and $G_{1}(t)=$ $(n-1) G(t)+n t G^{\prime}(t) \in C\left(P_{n}(D)\right)$. Using the same argument and induction, we can show that for every $k \in \mathbb{N}, f_{k}(x) \in C^{1}(D)$ and $G_{k}(t) \in C^{1}\left(P_{n}(D)\right)$, where the functions $f_{k}$ and $G_{k}$ are defined recursively by $f_{k+1}(x)=x f_{k}^{\prime}(x), k \geq 0, f_{0}(x)=f(x)$, and $G_{k+1}(t)=(n-1) G_{k}(t)+n t G_{k}^{\prime}(t), k \geq 0, G_{0}(t)=G(t)$. From the definition of $f_{k}$, we get

$$
f_{k}(x)=\sum_{j=1}^{k} a_{k, j} x^{j} f^{(j)}(x),
$$

where $a_{k, 1}=a_{k, k}=1$ and $a_{k+1, j}=a_{k, j-1}+j a_{k, j}, j=2, \ldots, k$. Since $0 \notin D$, we get $f^{(k)}(x) \in C(D)$ for every $k \geq 0$, that is, $f \in C^{\infty}(D)$.

We proceed by induction with respect to $n \in \mathbb{N}, n \geq 2$. For $n=2$ we have

$$
\frac{f\left(x_{2}\right)-f\left(x_{1}\right)}{x_{2}-x_{1}}=G\left(x_{1} x_{2}\right), \quad x_{1}, x_{2} \in D, x_{1} \neq x_{2},
$$

and $f \in C^{\infty}(D)$. Set $x_{1}=x$ and let $x_{2} \rightarrow x$. We get

$$
f^{\prime}(x)=G\left(x^{2}\right)=\frac{f\left(x^{2}\right)-f(1)}{x^{2}-1}, \quad x \in D \backslash\{1\} .
$$

We may assume that $1 \in D$, otherwise we consider $f(a x)$ instead of $f(x)$ for some $a \in D$. Set $g(t)=: f(1+t)$. Since $0 \in D(g)$, the domain of $g$, and $g \in C^{\infty}(D(g)), g$ has a power series representation

$$
g(t)=\sum_{k=0}^{\infty} g_{k} t^{k}, \quad t \in(-r, r)
$$

for small $r>0$. Relations (2.6), with $x=t+1$, and (2.7) yield

$$
\sum_{k=1}^{\infty} g_{k} k t^{k-1}=g^{\prime}(t)=\frac{\left(g\left(t^{2}+2 t\right)-g(0)\right)}{t^{2}+2 t}=\sum_{s=0}^{\infty} g_{s+1} t^{s}(t+2)^{s} .
$$


88 Characterization of a class of functions using divided differences

Comparing the coefficients of $t^{j}$ in (2.8) we obtain

$$
(j+1) g_{j+1}=\sum_{s=0}^{j}\left(\begin{array}{c}
s \\
j-s
\end{array}\right) 2^{2 s-j} g_{s+1}, \quad j \geq 0,
$$

or, equivalently, (with $v=s+1$ )

$$
\left(j+1-2^{j}\right) g_{j+1}=\sum_{\nu=2}^{j}\left(\begin{array}{c}
v-1 \\
j-v+1
\end{array}\right) 2^{2 v-j-2} g_{\nu}, \quad j \geq 2 .
$$

Equation (2.10) is nontrivial only if $j \geq 2$. This is so because adding to $g$ (or $f$ ) a linear function and multiplying $g$ (or $f$ ) by a constant does not change its properties. The binomial coefficients in (2.10) are not zero only if $v-1 \geq j-v+1$, that is, if $v \geq j / 2+1 \geq 2$. Then (2.10) implies that $g_{j}$ is a multiple of $g_{2}$ for every $j \geq 2$. In particular, if $g_{2}=0$ then $g$ (and hence $f$ ) is a linear function.

If $g_{2} \neq 0$ we may assume that $g_{2}=1$. In this case (2.10) implies $g_{j}=(-1)^{j}$ for every $j \geq 2$. This follows from (2.9) and the identity

$$
\sum_{s=1}^{j}\left(\begin{array}{c}
s \\
j-s
\end{array}\right) 2^{2 s-j}(-1)^{s-j}=j+1, \quad j \geq 2,
$$

which is the special case $\alpha=2, \beta=-1$ of the formula

$$
A_{j}(\alpha, \beta):=\sum_{s=0}^{j}\left(\begin{array}{c}
s \\
j-s
\end{array}\right) \alpha^{2 s-j} \beta^{j-s}=\sum_{k=0}^{j} \lambda_{1}^{k} \lambda_{2}^{j-k}, \quad j \geq 0,
$$

where $\alpha, \beta \in \mathbb{C}$ and $\lambda_{1,2}$ are the zeros of $\lambda^{2}-\alpha \lambda-\beta$. Hence, it is enough to verify (2.12). For $j=0$ and $j=1,(2.12)$ is obvious. Next, for $j \geq 1$

$$
\begin{aligned}
A_{j+1}(\alpha, \beta)= & \sum_{s=1}^{j+1}\left(\begin{array}{c}
s \\
j+1-s
\end{array}\right) \alpha^{2 s-j-1} \beta^{j+1-s} \\
= & \alpha \sum_{s=1}^{j+1}\left(\begin{array}{c}
s-1 \\
j-(s-1)
\end{array}\right) \alpha^{2(s-1)-j} \beta^{j-(s-1)} \\
& +\beta \sum_{s=1}^{j}\left(\begin{array}{c}
s-1 \\
j-1-(s-1)
\end{array}\right) \alpha^{2(s-1)-(j-1)} \beta^{(j-1)-(s-1)} \\
= & \alpha A_{j}(\alpha, \beta)+\beta A_{j-1}(\alpha, \beta) \\
= & \alpha \sum_{k=0}^{j} \lambda_{1}^{k} \lambda_{2}^{j-k}+\beta \sum_{k=0}^{j-1} \lambda_{1}^{k} \lambda_{2}^{j-1-k} \\
= & \sum_{k=0}^{j}\left(\lambda_{1}^{k+1} \lambda_{2}^{j-k}+\lambda_{1}^{k} \lambda_{2}^{j+1-k}\right)-\sum_{k=0}^{j-1} \lambda_{1}^{k+1} \lambda_{2}^{j-k} \\
= & \sum_{k=0}^{j+1} \lambda_{1}^{k} \lambda_{2}^{j+1-k},
\end{aligned}
$$


where we used the identities

$$
\left(\begin{array}{c}
s \\
j+1-s
\end{array}\right)=\left(\begin{array}{c}
s-1 \\
j-s+1
\end{array}\right)+\left(\begin{array}{c}
s-1 \\
j-s
\end{array}\right), \quad s=1, \ldots, j,
$$

$\lambda_{1}+\lambda_{2}=\alpha, \lambda_{1} \lambda_{2}=-\beta$, and induction with respect to $j$.

Since $g_{j}=(-1)^{j}, j \geq 2$, it follows from (2.7) that $g(t)=g_{2} /(1+t)+A t+B$ for $t \in(-r, r)$. We have proved that for every $x_{0} \in D$ there exists $r\left(x_{0}\right)>0$ such that $f(x)=c / x+a x+b,\left|x-x_{0}\right|<r\left(x_{0}\right)$. We have to show that the coefficients $a, b$, and $c$ are independent of $x_{0} \in D$. Let $I_{1}$ and $I_{2}$ be two open subinterval of $D$ such that

$$
f(x)=\frac{c_{v}}{x}+a_{\nu} x+b_{v}, \quad x \in I_{\nu}, v=1,2 .
$$

From (2.5) we get

$$
f[x, y]=\frac{\left(c_{2}+a_{2} y^{2}+b_{2} y\right) x-\left(c_{1}+a_{1} x^{2}+b_{1} x\right) y}{(y-x) x y}=G(x y), \quad x \in I_{1}, y \in I_{2} .
$$

Let $C=x_{0} y_{0}$ for some $x_{0} \in I_{1}$ and $y_{0} \in I_{2}$, and let $\gamma_{C}=\left(I_{1} \times I_{2}\right) \cap\{(x, y): x y=C\}$. Then

$$
f[x, y]=\frac{\left(c_{2}+a_{2} y^{2}+b_{2} y\right) C-\left(c_{1} y^{2}+a_{1} C^{2}+b_{1} C y\right)}{\left(y^{2}-C\right) C}=G(C), \quad(x, y) \in \gamma_{C} .
$$

Since $\gamma_{C}$ is a continuous curve, (2.17) implies that $\left(b_{2}-b_{1}\right) C=0, a_{2} C-c_{1}=G(C) C$, and $\left(c_{2}-a_{1} C\right) C=-G(C) C^{2}$. Using that $C \neq 0$ we get $b_{1}=b_{2}$ and $a_{1} C-c_{2}=$ $G(C) C=a_{2} C-c_{1}$, hence, $\left(a_{2}-a_{1}\right) C=c_{1}-c_{2}$. Unless $a_{1}=a_{2}$ we can choose $C \neq\left(c_{1}-c_{2}\right) /\left(a_{2}-a_{1}\right)$ and that choice would give us a contradiction. Thus $a_{1}=a_{2}$ and then $c_{1}=c_{2}$.

Now assume that Theorem 1.1 is true for some $n \geq 2$ and consider the case $n+1$. Let $x_{1} \in D$ be fixed. We define $\tilde{f}(x):=f\left[x_{1}, x\right]$. Let $\left\{x_{j}\right\}_{j=2}^{n+1} \subset D \backslash\left\{x_{1}\right\}$ be distinct numbers. From (1.2) and (1.3) with $\tilde{\omega}_{n}(x):=\prod_{k=2}^{n+1}\left(x-x_{k}\right)$, we obtain

$$
\begin{aligned}
\tilde{f}\left[x_{2}, \ldots, x_{n+1}\right] & =\sum_{j=2}^{n+1} \frac{f\left(x_{j}\right)-f\left(x_{1}\right)}{\left(x_{j}-x_{1}\right) \tilde{\omega}_{n}^{\prime}\left(x_{j}\right)}=\sum_{j=1}^{n+1} \frac{f\left(x_{j}\right)-f\left(x_{1}\right)}{\omega_{n+1}^{\prime}\left(x_{j}\right)} \\
& =f\left[x_{1}, \ldots, x_{n+1}\right]=G\left(x_{1} \prod_{j=2}^{n+1} x_{j}\right),
\end{aligned}
$$

where we also used that $f\left(x_{1}\right)\left[x_{1}, \ldots, x_{n+1}\right]=0$. Hence, $\tilde{f}$ satisfies the conditions of Theorem 1.1 for $n$ points. By the induction assumption

$$
f\left[x_{1}, x\right]=\tilde{f}(x)=\sum_{j=-1}^{n-1} \tilde{a}_{j}\left(x_{1}\right) x^{j}, \quad x \in D \backslash\left\{x_{1}\right\},
$$


or, equivalently,

$$
f(x)=f\left(x_{1}\right)+\left(x-x_{1}\right) \sum_{j=-1}^{n-1} \tilde{a}_{j}\left(x_{1}\right) x^{j}=: \sum_{j=-1}^{n} a_{j}\left(x_{1}\right) x^{j}, \quad x \in D \backslash\left\{x_{1}\right\} .
$$

Equation (2.20) is true for $x=x_{1}$ as well. Furthermore, (2.20) is unique in the sense that the coefficients $a_{j}\left(x_{1}\right)$ are independent of $x_{1} \in D$. Indeed,

$$
x f(x)=\sum_{j=0}^{n+1} a_{j-1}\left(x_{1}\right) x^{j}=\sum_{j=0}^{n+1} a_{j-1}\left(x_{2}\right) x^{j}, \quad x \in D
$$

implies $a_{j}\left(x_{1}\right)=a_{j}\left(x_{2}\right), j=-1, \ldots, n$, since a nonzero polynomial has finitely many zeros.

Now assume that $0 \in D$. Then $D_{1}:=D \backslash\{0\}$ is an open set. For every $n-1$ distinct numbers $\left\{x_{j}\right\}_{j=2}^{n} \subset D_{1}$, from (2.18) with $\tilde{f}(x):=f[0, x]$ and (1.3) we obtain

$$
\tilde{f}\left[x_{2}, \ldots, x_{n}\right]=f\left[0, x_{2}, \ldots, x_{n}\right]=G(0) .
$$

Then $\tilde{f}(x)$ satisfies the conditions of Theorem 1.1 on the set $D_{1}$ and $0 \notin D_{1}$. Therefore, $\tilde{f}(x)=(f(x)-f(0)) / x=a_{-1} / x+p(x), p(x) \in \mathscr{P}_{n-2}$. Since $G \in C(D),(1.3)$ implies $f \in C(D)$ and $a_{-1}=0$. Hence $f(x)=f(0)+x p(x) \in \mathscr{P}_{n-1}$.

The formula for $G(t)$ follows from the identities $x^{k}\left[x_{1}, \ldots, x_{n}\right]=\delta_{n-1, k}, k=0, \ldots$, $n-1$, and

$$
\frac{1}{x}\left[x_{1}, \ldots, x_{n}\right]=\frac{(-1)^{n+1}}{x_{1} \cdots x_{n}}, \quad n \geq 1 .
$$

The proof of Theorem 1.1 is complete.

Proof of Theorem 1.2. The proof of Theorem 1.2 follows the same arguments as the proof of Theorem 1.1 except that after verifying $f \in C^{1}(D)$ as in the proof of Theorem 1.1, we automatically obtain that $f \in C^{\infty}(D)$ via Cauchy's integral formula.

\section{Acknowledgement}

The author would like to thank Boris Shekhtman for proposing the problem and for helpful suggestions and comments.

\section{References}

[1] K. M. Andersen, A characterization of polynomials, Math. Mag. 69 (1996), no. 2, 137-142. MR 97d:12001. Zbl 853.39011.

[2] C. de Boor, A Practical Guide to Splines, Applied Mathematical Sciences, vol. 27, SpringerVerlag, New York, 1978. MR 80a:65027. Zbl 406.41003.

Plamen Simeonov: Department of Computer and Mathematical Sciences, University of Houston-Downtown, One Main Street Houston, TX 77002, USA

E-mail address: simeonov@dt.uh.edu 


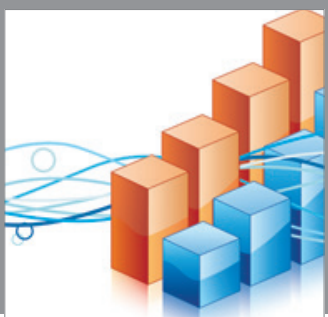

Advances in

Operations Research

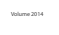

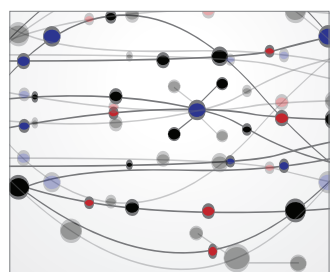

\section{The Scientific} World Journal
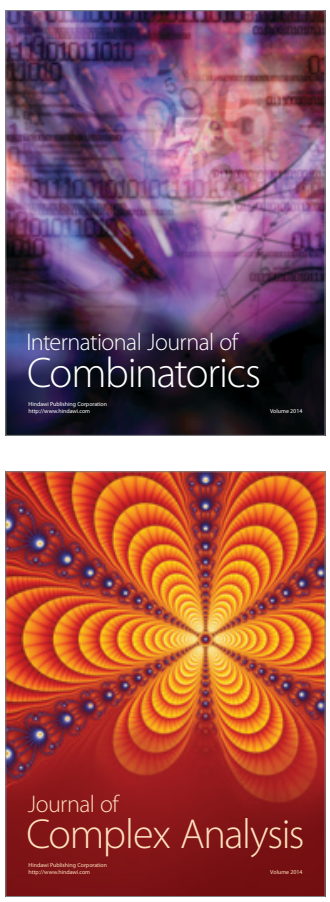

International Journal of

Mathematics and

Mathematical

Sciences
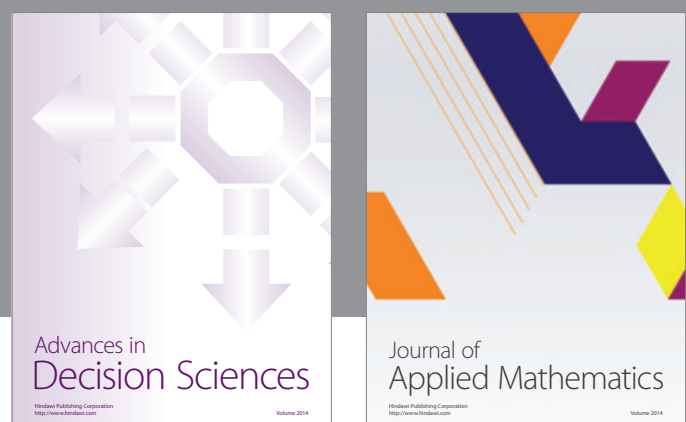

Journal of

Applied Mathematics
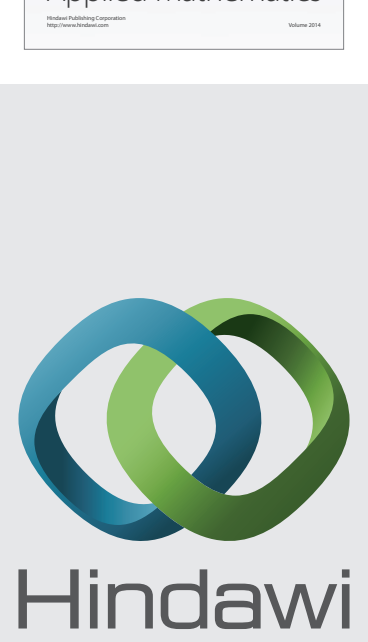

Submit your manuscripts at http://www.hindawi.com
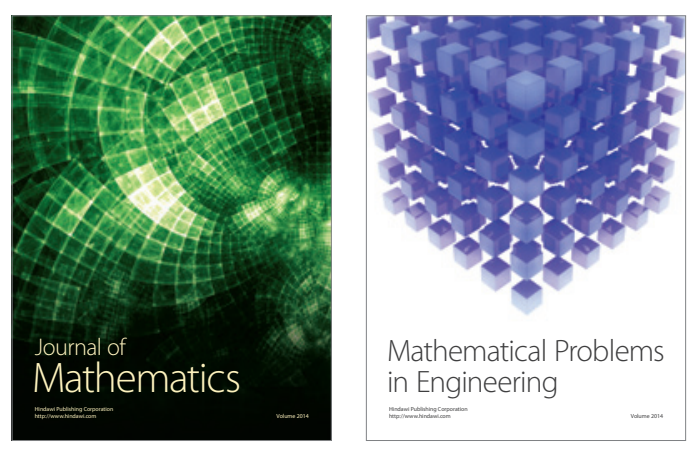

Mathematical Problems in Engineering
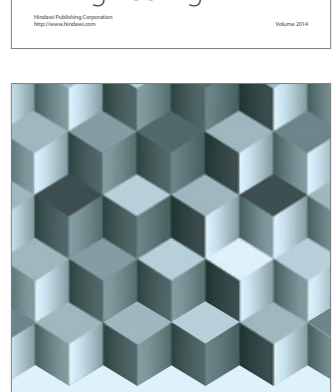

Journal of

Function Spaces
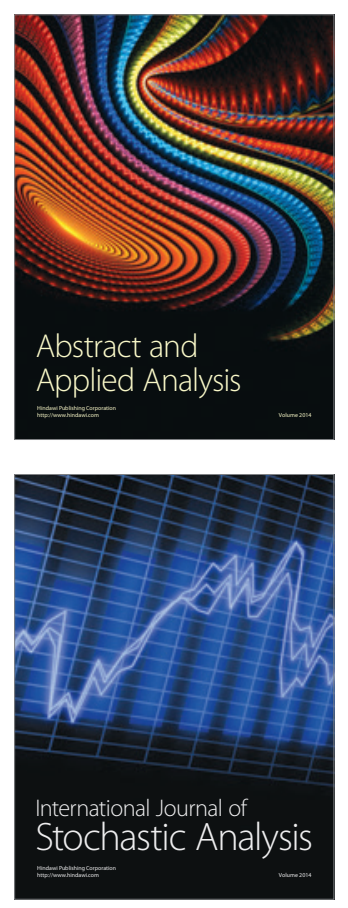

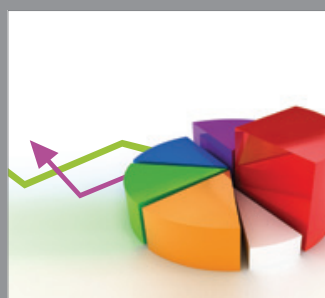

ournal of

Probability and Statistics

Promensencen
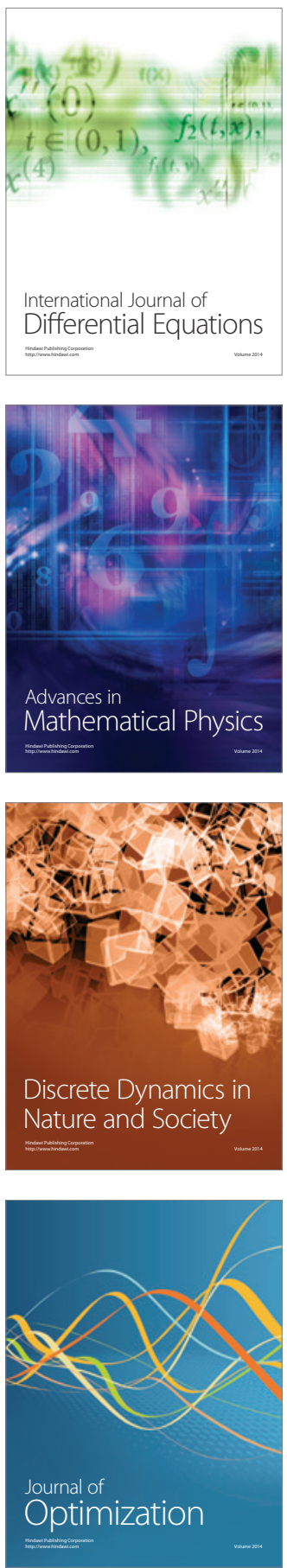